\title{
A IMPORTÂNCIA DA ATUAÇÃO DO FARMACÊUTICO NA FARMACOVIGILÂNCIA HOSPITALAR NO BRASIL: UMA REVISÃO DE LITERATURA
}

\section{THE IMPORTANCE OF THE PHARMACIST'S PERFORMANCE IN HOSPITAL PHARMACOVIGILANCE: A LITERATURE REVIEW}

\author{
Cristiane Mancio Sauma ${ }^{1}$, Ely de Nazaré Oliveira Drago ${ }^{1}$, Karla Suanny Navarro ${ }^{1}$, Marcella \\ Kelly Costa de Almeida ${ }^{1}$, Carla de Castro Sant' Anna ${ }^{1}$ \\ ${ }^{1}$ Universidade da Amazônia \\ E-mail para correspondência: santannacarla@yahoo.com.br \\ Submetido em: 08/10/2021 e aprovado em: 10/01/2022
}

\begin{abstract}
RESUMO
Introdução: Farmacovigilância é a ciência que estuda as atividades relacionadas a detecção, avaliação, compreensão e prevenção dos efeitos adversos a medicamentos. Investigando e notificando as queixas técnicas com base nas RAM's (Reações Adversas a Medicamentos), o farmacêutico é o profissional mais preparado e qualificado para reduzir os riscos relativos à utilização desses medicamentos, por meio do acompanhamento sistemático de ocorrências desses eventos. Objetivo: Demonstrar a importância da atuação do farmacêutico no âmbito da farmacovigilância hospitalar no Brasil. Método: Revisão de literatura, a partir de artigos encontrados nas bases de dados SciELO, LILACS, MedLine e Google Acadêmico, indexados no período de 2011 a 2021, por meio dos descritores: Farmacovigilância hospitalar; Atuação do Farmacêutico; Reações Adversas a Medicamentos (RAMs). As referências foram analisadas conforme ano, idioma, país de publicação e tipo de estudo e classificadas de acordo com seu foco em relação às atividades relacionadas a detecção, avaliação, compreensão e prevenção dos efeitos adversos a medicamentos (RAM's) e a atuação do Farmacêutico na Farmacovigilância Hospitalar. Resultados: A partir da leitura analítico-reflexiva de cerca de 1690 artigos indexados nas bases de dados, conforme os critérios de inclusão e exclusão, optou-se pela escolha daqueles que respondem à questão norteadora, restando 08 estudos. Utilizou-se duas categorias de investigação, ou seja, atividades relacionadas a detecção, avaliação, compreensão e prevenção dos efeitos adversos a medicamentos e atuação do Farmacêutico na Farmacovigilância Hospitalar. Conclusão: As publicações enfatizaram a discussão sobre a Farmacovigilância hospitalar e a atuação do farmacêutico direcionada à investigação e notificação de queixas com base nas RAM's, apresentando um impacto positivo dessa atuação em relação à assistência à saúde, como segurança do paciente. Tais estudos podem fundamentar o processo de tomada de decisão e a formulação de estratégias que visem ampliar a capacidade de gestão e a qualidade do serviço prestado.
\end{abstract}

Palavras-chave: Farmacovigilância hospitalar, reações adversas a medicamentos, farmacêutico. 


\begin{abstract}
Introduction: Pharmacovigilance is the science that studies activities related to detection, assessment, understanding and prevention of adverse drug effects. Investigating and notifying technical complaints based on ADRs (Adverse Reactions to Drugs), the pharmacist is the most prepared and qualified professional to reduce the risks related to the use of these drugs, through the systematic monitoring of the occurrence of these events. Objective: Demonstrate the importance of the role of the pharmacist in the context of hospital pharmacovigilance in Brazil. Method: Literature review, based on articles found in the SciELO, LILACS, MedLine and Academic Google databases, indexed from 2011 to 2021, using the descriptors: Hospital pharmacovigilance; Pharmacist's Performance; Adverse Drug Reactions (ADRs). References were analyzed according to year, language, country of publication and type of study and classified according to their focus in relation to activities related to detection, assessment, understanding and prevention of adverse drug effects (ADRs) and the role of the Pharmacist in Hospital Pharmacovigilance. Results: From the analytical-reflective reading of about 1690 articles indexed in the databases, according to the inclusion and exclusion criteria, it was decided to choose those that answered the guiding question, leaving 8 studies. Two categories of investigation were used, that is, activities related to detection, assessment, understanding and prevention of adverse drug effects and the role of the Pharmacist in Hospital Pharmacovigilance. Conclusion: The publications emphasized the discussion on hospital pharmacovigilance and the role of the pharmacist aimed at investigating and reporting complaints based on ADRs, presenting a positive impact of this role in relation to health care, such as patient safety. Such studies can support the decision-making process and the formulation of strategies aimed at expanding management capacity and the quality of the service provided.
\end{abstract}

Keywords: Hospital pharmacovigilance, adverse drug reactions, pharmaceutical.

\title{
INTRODUÇÃO
}

Os medicamentos são insumos indispensáveis na assistência farmacêutica, ferramentas com finalidade profilática, paliativa, curativa ou de diagnóstico, essenciais para garantir e melhorar a saúde, e a resolubilidade dos serviços de saúde ${ }^{1}$.

O uso de medicamentos mesmo de maneira correta não está isento de riscos, podendo trazer para o usuário alguns efeitos indesejados, ou até inesperados, acarretando complicações e causando danos que podem ser leves, prolongar o tempo de internação, ou até levar o mesmo a óbito ${ }^{2}$.

De acordo com a Organização Mundial de Saúde ${ }^{3}$, Reação Adversa a Medicamento (RAMs) é qualquer resposta prejudicial ou indesejável e não intencional, que ocorre com medicamentos em doses empregadas usualmente em seres humanos, para profilaxia, diagnóstico, tratamento de doença, ou para modificação de função fisiológica.

Atualmente, existe estudo sobre eventos adversos na área hospitalar, onde revisões sistemáticas sobre a ocorrência das RAMs, entre os pacientes internados, variam de 1,6\% a $41,4 \%$. 
As RAMs foram descritas como sendo responsável por até $10 \%$ das internações hospitalares nos diferentes países, e o custo das complicações associada à erros dos medicamentos implicam um custo de até $15 \%$ dos orçamentos hospitalares ${ }^{5}$.

Dados da $\mathrm{OMS}^{6}$ também mostram que mais de 50\% dos medicamentos são prescritos, administrados e vendidos indevidamente. Segundo o relatório publicado pelo Sistema Nacional de Informação Tóxico-Farmacológicas ${ }^{7}$, os medicamentos representaram 27,93\% dos casos de intoxicação, revelando a necessidade de informação e orientação dos pacientes para o uso racional de medicamentos.

São numerosos os erros na utilização dos medicamentos, sendo assim, aplicar a ferramenta de gerenciamento de riscos no estabelecimento pode ser a alternativa para o melhor controle e monitoramento dos processos ${ }^{8}$. Com isso, para que se possam prevenir riscos, é necessário identificar e analisar a origem do evento para que ações preventivas possam ser aplicadas antes da ocorrência e não após o dano? ${ }^{9}$

A farmacovigilância tem como objetivo o mapeamento e rastreamento das RAM, com o monitoramento desses dados visando a redução dos eventos adversos realizando um processo bem planejado, o farmacêutico pode utilizar ferramentas e mecanismos como os POP'S (procedimentos operacionais padrão) para facilitar este processo ${ }^{10}$.

Nesse contexto, o papel do farmacêutico no processo da farmacovigilância hospitalar é de suma importância, pois é o profissional mais bem preparado e qualificado para tratar do assunto, já que o mesmo precisará discorrer por vários processos de gerenciamento da área hospitalar que vão desde aquisição, recebimento, guarda, até a dispensação desse medicamento $^{11}$.

A ausência de profissionais capacitados por falta de iniciativas governamentais, além de uma política de saúde irregular e inconstante, prejudica a adequada orientação sobre o correto uso de medicamentos e contribui com a manutenção de índices elevados de intoxicações. Técnicas de marketing atraem usuários e prescritores, e favorecem a utilização indevida de medicamentos por uma parcela importante da população ${ }^{12}$.

O farmacêutico clínico, tem como principal função garantir o uso seguro dos medicamentos prescritos para os pacientes, com foco em melhorar os desfechos clínicos e reduzir eventos adversos a medicamentos. Porém, os principais desafios são a integração com a equipe multiprofissional, desenvolvimento de trabalhos científicos que demonstrem os resultados obtidos com a atuação do farmacêutico clínico e a necessidade de recursos humanos 
e tecnológicos, pois, na maioria dos hospitais brasileiros, ainda se tem um número elevado de leitos por farmacêutico e a falta de sistemas informatizados para auxiliar no serviço ${ }^{13}$.

Dessa forma, o objetivo deste artigo foi demonstrar, por meio de dados científicos, a importância da atuação do farmacêutico no âmbito da farmacovigilância hospitalar, contribuindo de forma positiva para a diminuição dos casos de intoxicação medicamentosa.

\section{MÉTODOS}

No processo de definição do tema e questão de pesquisa da revisão de literatura, elaborou-se uma pergunta de pesquisa a qual norteou a condução do estudo: "Quais as evidências científicas sobre a importância da atuação do farmacêutico no âmbito da farmacovigilância hospitalar?".

Foi realizada uma revisão integrativa de literatura sobre a importância do farmacêutico no acompanhamento da farmacovigilância no âmbito hospitalar, baseando-se em artigos científicos indexados. O estudo teve como objetivo a busca por dados científicos na demonstração da importância da atuação do farmacêutico no âmbito da farmacovigilância hospitalar, contribuindo de forma positiva para a diminuição dos casos de intoxicação medicamentosa.

Os dados foram obtidos através de pesquisas da literatura através de bases de dados: Scientific Electronic Library Online (SciELO); Medical Literature Analysis and Retrieval System Online (MEDLINE); Lilacs (Latin American and Caribbean Literature in Health Sciences) e o diretório Google Acadêmico (Google Scholar), utilizando os artigos científicos a respeito do assunto que fossem encontrados nos idiomas inglês e português e que respondessem à questão norteadora.

A coleta de dados deu-se a partir dos anos de 2011 a 2021, para retratar a atuação do farmacêutico na farmacovigilância hospitalar. Os descritores (DeCS) utilizados foram: Farmacovigilância hospitalar (Hospital pharmacovigilance); Atuação do Farmacêutico (Pharmacist's Performance); Reações Adversas a Medicamentos (RAMs) (Adverse Drug Reactions, ADRs).

Quanto aos critérios de inclusão, foram utilizados todos os tipos de desenho de estudos retrospectivos e prospectivos que abordassem sobre a importância do farmacêutico no acompanhamento da farmacovigilância no âmbito hospitalar, publicados em português com texto disponível em revistas indexadas nas bases de dados citadas. Além destes, foram incluídos estudos multiprofissionais. Foram excluídos artigos duplicados, relato de experiência, revisão 
sistemática de literatura, obras literárias, trabalhos de graduação, artigos publicados em revistas não indexadas em banco de dados selecionados, artigos fora do período de seleção.

Para garantir a validade da revisão, a análise crítica dos estudos foi iniciada a partir da categorização, ordenação e sumarização dos resultados. Assim, os dados obtidos durante a coleta de dados foram armazenados em planilha do programa Excel (Microsoft Excel, 2019), com o objetivo de analisar; demonstrar e comparar os resultados positivos e negativos do papel do farmacêutico com a implementação do serviço de farmacovigilância hospitalar, gerando assim, gráficos, tabelas e fluxogramas.

A apresentação da revisão de literatura aconteceu de forma clara e concisa para permitir ao leitor avaliar criticamente os resultados. Na revisão de literatura os estudos foram reunidos em categorias temáticas agrupadas por semelhança de conteúdo, e os resultados interpretados com base na literatura correlata ao tema da pesquisa. Continham, então, informações especificadas e pertinentes, com base em metodologias contextualizadas, sem omitir qualquer evidência relacionada.

Foram considerados os aspectos éticos, mantendo as ideias e conceitos originais dos autores pesquisados, citando-os e referenciando-os dentro das normas da Associação Brasileira de Normas Técnicas (ABNT). Por se tratar de um estudo bibliográfico, não há relação direta com seres humanos ou animais, conforme a Resolução 466/2012, do Conselho Nacional de Saúde/MS, que dispõe sobre pesquisa envolvendo seres humanos. Deste modo, não houve a necessidade de encaminhar a presente pesquisa ao Comitê de Ensino e Pesquisa.

\section{RESULTADOS}

A pesquisa nas bases de dados realizada pela Biblioteca Virtual de Saúde (BVS), a partir dos DeCS, resultou em cerca de 776 artigos na base SciELO, 180 na LILACS; 305 na MedLine; e, 429 no Google Acadêmico, totalizando 1.690 artigos científicos.

Conforme a aplicação do critério de inclusão descrito no método, após a leitura dos resumos/íntegra, selecionaram-se 87 artigos na base SciELO; 82 na MedLine; 80 na LILACS e 16 no Google Acadêmico. Todavia, dentre estes artigos, poucos responderam à questão norteadora, além da ocorrência de artigos duplicados, restando 04 na SciELO; 01 na MedLine; 02, na LILACS; e, 01 no Google Acadêmico.

Por fim, restaram 08 artigos que constituíram a amostra da revisão, considerando os dados expostos na figura 1 . 
Figura 1 - Fluxograma do processo de busca e seleção dos artigos da revisão, 2021.

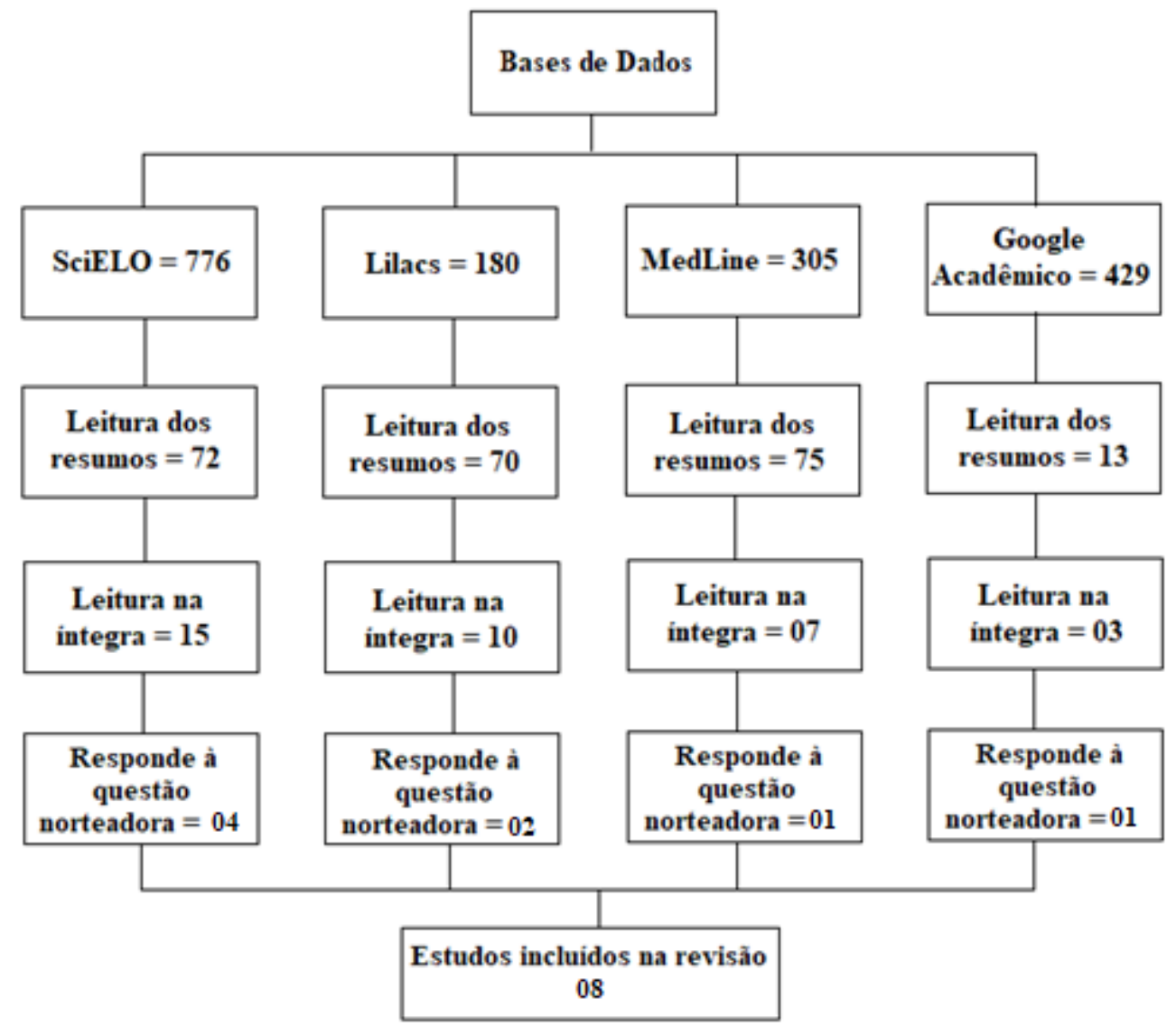

Fonte: Autoras, 2021.

No quadro 1, consta os artigos, conforme as seguintes variáveis: título/autor, ano, periódico/base e objetivo(s). Estas variáveis foram utilizadas para levantamento dos dados, comumente empregado em Revisões de Literatura.

Quadro 1 - Caracterização dos artigos selecionados para análise, segundo ordem, título/autor, ano, periódico/base e objetivos (Brasil, 2011 a 2021)

\begin{tabular}{|c|c|c|c|c|}
\hline Ordem & Título/Autor & Ano & Periódico/Base & Objetivo(s) \\
\hline F1 & $\begin{array}{c}\text { Notificação de reações } \\
\text { adversas em um Hospital } \\
\text { Sentinela de Fortaleza, } \\
\text { Ceará. ROMEU, G. A.; } \\
\text { TÁVORA, M. R. F.; } \\
\text { COSTA, A. K. M.; SOUZA, } \\
\text { M. O. B.; GONDIM, A. P. } \\
\text { S. }\end{array}$ & $2011^{14}$ & $\begin{array}{l}\text { Rev. Bras. Farm. Hosp. } \\
\text { Serv. Saúde/SciELO }\end{array}$ & $\begin{array}{l}\text { Descrever as notificações de } \\
\text { suspeitas de reações } \\
\text { adversas a medicamentos de } \\
\text { um hospital sentinela de } \\
\text { Fortaleza, estado do Ceará. }\end{array}$ \\
\hline $\mathrm{F} 2$ & $\begin{array}{c}\text { Notificações de } \\
\text { farmacovigilância em um } \\
\text { Hospital Oncológico } \\
\text { Sentinela da Paraíba. } \\
\text { DUARTE, M. L.; }\end{array}$ & $2014^{15}$ & $\begin{array}{l}\text { Rev. Bras. Farm. Hosp. } \\
\text { Serv. Saúde/SciELO }\end{array}$ & $\begin{array}{c}\text { Identificar e avaliar os } \\
\text { registros de notificações de } \\
\text { farmacovigilância durante } \\
\text { cinco anos em um hospital } \\
\text { oncológico sentinela da } \\
\text { Paraíba. }\end{array}$ \\
\hline
\end{tabular}




\begin{tabular}{|c|c|c|c|c|}
\hline & $\begin{array}{c}\text { BATISTA, L. M.; } \\
\text { ALBUQUERQUE, P. M. S. }\end{array}$ & & & \\
\hline F3 & $\begin{array}{c}\text { Atividades de } \\
\text { farmacovigilância em um } \\
\text { hospital pediátrico: uma } \\
\text { proposta de melhoria. } \\
\text { FERREIRA NETO, P. T. P.; } \\
\text { SIMÃO, R. F. M.; } \\
\text { LACAVA, A. M. }\end{array}$ & $2014^{16}$ & Revista ACRED/Lilacs & $\begin{array}{c}\text { Analisar as atividades de } \\
\text { farmacovigilância em um } \\
\text { hospital pediátrico privado, à } \\
\text { luz das diretrizes para } \\
\text { criação e funcionamento de } \\
\text { um Centro de } \\
\text { Farmacovigilância da } \\
\text { Organização Mundial da } \\
\text { Saúde (OMS). }\end{array}$ \\
\hline F4 & $\begin{array}{c}\text { Farmacovigilância } \\
\text { hospitalar: importância do } \\
\text { treinamento de profissionais } \\
\text { na potencialização de suas } \\
\text { ações. PEZATO, T. P. J.; } \\
\text { CESARETTI, M. L. R. }\end{array}$ & $2015^{17}$ & $\begin{array}{l}\text { Rev. Fac. Ciênc. } \\
\text { Méd/Google } \\
\text { Acadêmico }\end{array}$ & $\begin{array}{c}\text { Identificar e caracterizar as } \\
\text { notificações de reações, } \\
\text { eventos adversos e desvios } \\
\text { de qualidade de } \\
\text { medicamentos em hospital } \\
\text { privado e treinar } \\
\text { profissionais da saúde sobre } \\
\text { farmacovigilância. }\end{array}$ \\
\hline F5 & $\begin{array}{c}\text { Reações Adversas a } \\
\text { Medicamentos e } \\
\text { Farmacovigilância: } \\
\text { Conhecimentos e Condutas } \\
\text { de Profissionais de Saúde de } \\
\text { um Hospital da Rede } \\
\text { Sentinela. MODESTO, A. } \\
\text { C. F.; FERREIRA, T. X. A. } \\
\text { M.; PROVIN, M. P.; } \\
\text { AMARAL, R. G.; LIMA, D. } \\
\text { M. }\end{array}$ & $2016^{18}$ & $\begin{array}{c}\text { Revista Brasileira de } \\
\text { Educação } \\
\text { Médica/SciELO }\end{array}$ & $\begin{array}{l}\text { Identificar o conhecimento e } \\
\text { as condutas dos profissionais } \\
\text { de saúde de um hospital de } \\
\text { ensino em relação às reações } \\
\text { adversas a medicamentos e à } \\
\text { farmacovigilância e se há } \\
\text { associação com o seu perfil } \\
\text { profissiográfico. }\end{array}$ \\
\hline F6 & $\begin{array}{c}\text { Desenvolvimento de } \\
\text { instrumento para avaliação } \\
\text { de notificações de suspeita } \\
\text { de eventos adversos a } \\
\text { medicamentos. PEREIRA, } \\
\text { L. M. V.; MENEZES, J. C.; } \\
\text { VENDRUSCOLO, A. C. S. }\end{array}$ & $2016^{19}$ & $\begin{array}{c}\text { Revista } \\
\text { QualidadeHQ/MedLine }\end{array}$ & $\begin{array}{c}\text { Desenvolver uma ficha para } \\
\text { nortear as avaliações das } \\
\text { notificações espontâneas } \\
\text { recebidas pelo Serviço de } \\
\text { Gerenciamento de Riscos do } \\
\text { Hospital das Clínicas da } \\
\text { Faculdade de Medicina de } \\
\text { Ribeirão Preto - } \\
\text { HCRP/USP. }\end{array}$ \\
\hline F7 & $\begin{array}{c}\text { Análise das ocorrências de } \\
\text { incidentes relacionados aos } \\
\text { medicamentos } \\
\text { potencialmente perigosos } \\
\text { dispensados em hospital de } \\
\text { ensino. BASILE, L. C.; } \\
\text { SANTOS, A.; STELZER, L. } \\
\text { B.; ALVES, R. C.; } \\
\text { FONTES, C. M. B.; } \\
\text { BORGATO, M. H. et al. }\end{array}$ & $2019^{20}$ & $\begin{array}{c}\text { Rev Gaúcha } \\
\text { Enferm./Lilacs }\end{array}$ & $\begin{array}{c}\text { Analisar as notificações de } \\
\text { incidentes relacionados aos } \\
\text { medicamentos } \\
\text { potencialmente perigosos } \\
\text { dispensados em um hospital } \\
\text { de ensino do interior de São } \\
\text { Paulo. }\end{array}$ \\
\hline
\end{tabular}




\begin{tabular}{|c|c|c|c|c|}
\hline F8 & $\begin{array}{c}\text { Retrospectiva de 20 anos de } \\
\text { atividade da Unidade de } \\
\text { Farmacovigilância do Porto, } \\
\text { Portugal. FERREIRA-DA- } \\
\text { SILVA, R. et al. }\end{array}$ & & $\begin{array}{c}\text { Cadernos de Saúde } \\
\text { Pública/ SciELO }\end{array}$ & $\begin{array}{c}\text { Caracterizar as reações } \\
\text { adversas a medicamentos } \\
\text { recebidas pela Unidade de } \\
\text { Farmacovigilância do Porto } \\
\text { (UFPorto), Portugal, ao } \\
\text { longo de duas décadas de } \\
\text { atividade. }\end{array}$ \\
\hline
\end{tabular}

Fonte: Das autoras, 2021.

Dentre os 08 artigos encontrados nessa produção, 01 (um) foi publicado em 2011 (F1), 02 (dois) em 2014 (F2, F3), 01 em 2015 (F4), 02 em 2016 (F5, F6), 01 (um) em 2019 (F7), 01 (um) em 2021 (F8). Corrobora-se, portanto, a afirmação de que os estudos escolhidos foram publicados em bases de dados dos últimos 10 anos (2011 a 2021).

Em detrimento às revistas onde os artigos foram publicados, identificou-se que 02 (dois) artigos foram publicados na Revista Brasileira de Farmacologia Hospitalar e Serviços de Saúde. Os artigos restantes foram publicados em revistas distintas, tendo como exemplo, a Revista Brasileira de Educação Médica, a Revista Qualidade HQ, o Caderno de Saúde Pública, dentre outras de igual relevância.

No quadro 2, são demonstradas as metodologias empregadas nos referidos artigos, conferindo em sua maioria estudos de base descritiva e retrospectiva, conforme expresso nos critérios de inclusão.

Quadro 2 - Metodologia empregada dos artigos selecionados.

\begin{tabular}{|c|l|}
\hline Ordem & \multicolumn{1}{|c|}{ Metodologia } \\
\hline F1 & $\begin{array}{l}\text { Estudo descritivo e retrospectivo que utilizou, como fonte secundária, a ficha de notificação de } \\
\text { suspeita de reação adversa a medicamentos do sistema de farmacovigilância do ano de 2007. }\end{array}$ \\
\hline F2 & $\begin{array}{l}\text { Estudo de caráter documental, transversal e retrospectivo que utilizou como fonte de dados a ficha } \\
\text { de notificação de suspeita de reação adversa a medicamento (RAM) e de queixa técnica sobre } \\
\text { medicamentos do serviço de Gerência de Risco do hospital no período de 01 de janeiro de 2008 a 31 } \\
\text { de dezembro de 2012. }\end{array}$ \\
\hline F3 & $\begin{array}{l}\text { Análise de documentos (subnotificações) provenientes de um hospital privado, exclusivamente } \\
\text { pediátrico, localizado na zona norte da capital do Estado do Rio de Janeiro. A subnotificação foi } \\
\text { quantificada e calculada pela relação entre o número de efeitos realmente observados pelos } \\
\text { profissionais de saúde e aqueles relatados espontaneamente ao sistema de farmacovigilância. }\end{array}$ \\
\hline F4 & $\begin{array}{l}\text { Estudo seccional e quantitativo de base hospitalar, conduzido em um hospital privado no município } \\
\text { de Sorocaba, no Estado de São Paulo, no período de maio a novembro de 2013. As notificações sobre } \\
\text { reações adversas, eventos adversos a medicamentos e desvios de qualidade encontram-se disponíveis } \\
\text { no sistema informatizado e integrado do hospital. }\end{array}$ \\
\hline F5 & $\begin{array}{l}\text { Estudo transversal, utilizando-se um instrumento validado que aborda questões sobre caracterização } \\
\text { da população estudada, conhecimentos e condutas. }\end{array}$ \\
\hline F6 & $\begin{array}{l}\text { Levantamento bibliográfico considerando a temática farmacovigilância; após, foram extraídos os } \\
\text { principais pontos para a análise de notificações envolvendo medicamentos, conforme ficha de } \\
\text { avaliação de suspeita de evento adverso a medicamento (EAM) foram considerados os dados } \\
\text { exigidos pela ANVISA para preenchimento do NOTIVISA descritos nas diretrizes para o } \\
\text { Gerenciamento do Risco em Farmacovigilância. }\end{array}$ \\
\hline
\end{tabular}




\begin{tabular}{|c|l|}
\hline F7 & $\begin{array}{l}\text { Estudo descritivo, retrospectivo com abordagem quantitativa dos dados provenientes da análise das } \\
\text { notificações em farmacovigilância que ocorreram no período de janeiro de } 2009 \text { a dezembro de 2014, } \\
\text { a partir de tabelas e gráficos, apresentando as frequências absolutas/relativas. }\end{array}$ \\
\hline F8 & $\begin{array}{l}\text { Este estudo, observacional, retrospectivo e descritivo, considerou todas as notificações de suspeitas } \\
\text { de reações adversas a medicamentos que foram reportadas à UF Porto desde janeiro de } 2001 \text { e } \\
\text { dezembro de 2019. Foram incluídas as notificações que verificaram os critérios mínimos de } \\
\text { validação, designadamente: doente, notificador, medicamento(s) suspeito(s) e evento(s) adverso(s). }\end{array}$ \\
\hline
\end{tabular}
Fonte: Das autoras, 2021.

No que se refere aos sujeitos investigados, todas as publicações utilizaram farmacêuticos como participantes da pesquisa. Ao tratar sobre a temática todas as publicações são direcionadas à atuação deste profissional na área de farmacovigilância hospitalar.

Quanto à localidade de realização/publicação das pesquisas, observou-se uma diversificação no espaço brasileiro, ou seja, os estudos seguiram critérios regionais, conforme: Sul, Nordeste, Sudeste, Centro Oeste. De forma complementar, um dos estudos foi realizado em Portugal.

Dos artigos pesquisados, portanto, houve maior ênfase para os Estados da região sudeste. Destaca-se que alguns artigos referentes à temática em questão foram encontrados na Região Norte, contudo, além de não responderem à questão norteadora, pertencem a anos anteriores aos escolhidos para as bases de dados (critério de exclusão), havendo a necessidade de descartá-los.

Conforme a análise e a complexidade dos artigos científicos, originaram-se duas categorias de resultados, que permitiram uma melhor apresentação das evidências científicas sobre a função do profissional de farmácia na farmacovigilância hospitalar. São elas:

Categoria 1 - Atividades relacionadas a detecção, avaliação, compreensão e prevenção dos efeitos adversos a medicamentos (RAM's)

Há que se frisar a notificação espontânea de RAM, pois esta constitui a principal fonte de informação em farmacovigilância ${ }^{14}$. Contudo, tem-se observado variações globais ao longo dos anos, sendo resultado da alteração do paradigma da farmacovigilância nas últimas duas décadas. Foi observada uma mudança na consciência da farmacovigilância como uma simples atividade de recolha e tratamento de suspeitas de reações adversas a medicamentos, para uma abordagem mais complexa e proativa na gestão do risco ${ }^{21}$.

Se o ambiente hospitalar representa um local propício para a ocorrência de falhas e acidentes decorrentes do uso de tecnologias de saúde, constata-se a necessidade de mais investimento nessa área, principalmente em relação à sensibilização da importância da notificação para obtenção de dados para fins de regulamentação sanitária e garantia da segurança do usuário, prevenindo as RAMs ${ }^{15}$. 
Essa prevenção perpassa inicialmente pela caracterização do evento adverso. É importante conhecer se a manifestação ocorreu quando o paciente estava internado, ou em procedimento ambulatorial, ou ainda, se não estava no hospital. O local da ocorrência impacta na qualidade dos dados levantados e a redução dos eventos adversos precisa ocorrer por meio do uso de métodos eficazes para a detecção e avaliação. Alguns fatores podem favorecer a ocorrência destes eventos, como as propriedades do próprio fármaco ou as características do paciente (idade, gênero, patologias associadas) e o uso da polifarmácia ${ }^{19}$.

Outros fatores são o trabalho exaustivo dos profissionais de saúde no que se refere às notificações. É um inconveniente para o relato de RAMs, pois além da prestação da assistência, envolvimento com os familiares e os afazeres rotineiros, existe a rotina de notificação que requer uma atenção e dedicação no preenchimento das RAMs ${ }^{15}$. Assim, quando há um número de notificações expressivo, tem-se a necessidade de adoção de estratégias a fim de garantir maior segurança do paciente ${ }^{20}$.

Essa garantia perpassa pelo monitoramento dos eventos adversos e observância de queixas técnicas, com a adoção de medidas como barreiras que reduzam a ocorrência dos erros (identificação dos medicamentos potencialmente perigosos ao serem dispensados às unidades hospitalares), utilização de protocolos e padronização da comunicação sobre os tratamentos, fornecimento e melhoria do acesso à informação, centralização dos processos de maior risco de erros, incorporação de alertas automáticos nos sistemas informatizados, uso de procedimentos de dupla conferência dos medicamentos, dentre outros ${ }^{20}$.

Saliente-se que o incentivo à notificação de RAM é considerado elemento chave para o fortalecimento dos sistemas de monitorização, utilizado pela farmacovigilância, sendo de suma importância para a melhoria da qualidade e do uso racional dos medicamentos ${ }^{14}$.

\section{Categoria 2 - Atuação do Farmacêutico na Farmacovigilância Hospitalar}

Desde 1963 que a farmacovigilância foi reconhecida como uma área prioritária na saúde pública a nível global pela Organização Mundial da Saúde (OMS), garantindo a monitorização permanente da segurança dos medicamentos ${ }^{21}$.

Há necessidade de investimentos em sistemas de farmacovigilância, que consiste na ciência e nas atividades de detecção, avaliação, compreensão e prevenção de efeitos adversos ou qualquer outro problema relacionado ao medicamento. O principal método para obtenção de informações de eventos adversos a medicamentos é a notificação voluntária de $\operatorname{casos}^{19}$. 
A atuação na farmacovigilância hospitalar é relevante, porém destaca-se a necessidade de uma capacitação contínua, ou seja, um reforço positivo na compreensão do assunto trabalhado, na intervenção educativa com estímulo à notificação, mudança de cultura e melhora da assistência ao paciente ${ }^{17}$, até porque a formação do profissional influencia seu conhecimento e condutas frente às reações adversas a medicamentos ${ }^{18}$.

Essa corresponsabilidade deve-se ao apoio ao desenvolvimento da farmacologia clínica na unidade de saúde, levando em consideração o envolvimento de associações profissionais, a participação em conferências científicas, a publicação dos achados em periódicos e a retroalimentação, ou seja, a devolução da informação processada ao profissional notificador ${ }^{16}$.

Outro cuidado importante é a facilidade de acesso aos formulários, que deverão estar ao alcance de todos os profissionais de quem se espera a notificação. Este acesso previne a subnotificação, que tem por causas a falta de conhecimentos, percepção e compreensão dos incidentes ${ }^{16}$.

Durante a ocorrência/suspeita de uma RAM, cabe ao profissional farmacêutico comunicar ao profissional médico e registrar a suspeita no prontuário do paciente. Para que isto ocorra, além de possuir extensa formação em farmacologia e toxicologia, precisa relacionar a notificação de RAM como parte de suas obrigações profissionais (gerenciamento) ${ }^{20}$.

Entre os profissionais de saúde, o médico é aquele que mais notifica as suspeitas de reações adversas a medicamentos, seguindo-se o farmacêutico e, por fim, o enfermeiro ${ }^{21}$. Portanto, diante de uma notificação de evento adverso, relacionado a suspeita RAMs ou inefetividade terapêutica, cabe ao farmacêutico realizar a busca de referências na literatura e, após a análise, garantir informações suficientes para que seja possível registrá-la no banco de dados da Agência Nacional de Vigilância Sanitária - NOTIVISA ${ }^{19}$.

Assim, a sistematização de informações é importante para avaliação das notificações de suspeitas de eventos adversos envolvendo medicamentos e da existência de fatores que podem favorecer a ocorrência dos mesmos e interferir no resultado da análise pelo farmacêutico, o desenvolvimento de uma ferramenta que direcione a coleta de dados é de fundamental importância para otimização da investigação e confiabilidade do seu resultado ${ }^{19}$.

Reforça-se a necessidade de capacitação como estratégia de sensibilização do profissional para notificação espontânea, procurando demonstrar que essa notificação não se traduz em erro de prescrição ${ }^{14}$. 


\section{DISCUSSÃO}

A apresentação das descrições metodológicas dos estudos incluídos revela, no que se refere à atuação do farmacêutico na farmacovigilância hospitalar, que há um predomínio de estudos qualitativos, descritivos e de cunho observacional analítico.

Os aspectos de iniciativas que podem ser implantadas pelo farmacêutico para melhoria do estado de saúde da comunidade estão relacionados ao acompanhamento e educação do paciente e para o paciente, a avaliação dos seus fatores de risco, prevenção das doenças, promoção da saúde e vigilância das doenças ${ }^{22}$.

Neste novo contexto da prática farmacêutica, no qual a preocupação com o bem-estar do paciente, somando esforços com outros profissionais de saúde e aos da comunidade para a promoção de saúde, o farmacêutico assume um papel de fundamental importância ${ }^{23}$.

$\mathrm{Na}$ era da medicina moderna, o uso preciso de medicamentos consiste na principal medida que liga a equipe multidisciplinar em atividades no cuidado com o paciente. Portanto, o uso racional continua configurando como uma das prioridades na saúde pública mundial ${ }^{24}$.

A idéia de que os medicamentos têm implícita a possibilidade de induzir efeitos adversos (RAMs) parece estar bem estabelecida entre a comunidade clínica e de usuários. Se a decisão terapêutica terá sempre de ponderar os "benefícios" esperados do medicamento, assim como os "riscos" da sua utilização, então a relação destes dois termos resulta um conceito mais robusto, ou seja, a segurança do medicamento. O peso dado a cada uma destas definições é relativo e variável entre patologias e doentes ${ }^{21}$.

Quando ocorre o uso irracional de medicamentos, uma de suas causas é devido à falta de orientação de um profissional habilitado, ocasionando vários casos de intoxicação. A notificação aos órgãos do Ministério da Saúde é de grande relevância, principalmente pela compreensão do impacto clínico no tratamento de doenças com as medicações e da pressão seletiva a ser exercida durante o uso, consistem em elementos fundamentais para a otimização de estratégias para a prescrição de medicamentos ${ }^{25}$.

Deste modo, as reações adversas a medicamentos (RAM) são um dos graves problemas de saúde pública em todo o mundo, responsáveis por numerosas hospitalizações, pelo aumento do tempo de permanência hospitalar e, até mesmo, por óbitos. Estudos internacionais evidenciam que elas representam a quarta causa de óbito nos EUA e são responsáveis por cerca de 3 a 6\% das hospitalizações. Estudos relatam a prevalência de 10 a 30\% dessas reações durante a hospitalização ${ }^{1}$. 
Quanto à farmacovigilância, a Organização Mundial da Saúde a define como o conjunto de atividades relativas à detecção, avaliação, compreensão e prevenção de efeitos adversos ou outros problemas relacionados a medicamentos. Para que um programa de farmacovigilância tenha êxito é necessário um bom nível de informação dos profissionais de saúde acerca da reação adversa, eventos adversos e desvio de qualidade de medicamentos. As atividades de minimização do risco para serem eficazes, requerem comunicação com/entre pacientes e profissionais de saúde ${ }^{26}$.

Cabe ao Sistema de Farmacovigilância, além da identificação de RAM, a disponibilização de informações e orientações atualizadas, claras e imparciais sobre medicamentos, tornando-as acessíveis ao usuário e aos profissionais de saúde; e normatização de ações preventivas aos riscos dessas reações, de modo a contribuir para a melhoria contínua da qualidade da assistência prestada ao paciente ${ }^{1}$.

Assim, na farmacovigilância o farmacêutico identifica e avalia os efeitos, agudos ou crônicos, dos riscos do uso dos tratamentos farmacológicos no âmbito hospitalar ou prestando serviços em farmácias comunitárias em usuários dos sistemas de atenção à saúde 27.

Muitos profissionais deixam de notificar por insegurança e notificam apenas os casos em que se observa uma relação causal, desconhecem o que notificar e são complacentes, pois creem que todos os eventos adversos a medicamentos (EAM) foram identificados na etapa da pesquisa clínica. Estudos explicam que os profissionais de saúde não estão devidamente sensibilizados e motivados acerca da farmacovigilância, uma vez que ainda não são devidamente elucidados os conceitos, a importância e as práticas envolvidas neste serviço ${ }^{26}$.

Ressalta-se que é preciso uma educação permanente para a equipe multiprofissional devido à grande negligência mediante os casos que precisam ser notificados. Destacando como principal profissional responsável por incentivar a notificação em farmacovigilância o farmacêutico clínico garantindo a qualidade de assistência prestada ao paciente adequando a sua aplicação à saúde individual e coletiva ${ }^{26}$.

Portanto, a atuação do farmacêutico dentro da farmacovigilância hospitalar precisa estar em consonância com a saúde pública; é um trabalho que deve ser valorizado como essencial para a aplicação de técnicas que possam subsidiar as prescrições médicas recebidas no serviço e avaliadas de forma correta para estabelecer um sistema de alerta permanente para a detecção de RAM's'.

Considerando-se as limitações do presente estudo, observou-se que há uma preocupação no meio científico em destacar a participação efetiva do profissional farmacêutico na 
farmacovigilância hospitalar. Deste modo, surge a necessidade de maiores investimentos em cursos de capacitação (educação contínua) na área.

Outra limitação ocorreu durante a análise e coleta de artigos direcionados ao presente estudo, no qual foi observado um quantitativo baixo de artigos atualizados que respondessem à questão norteadora, promovendo certa dificuldade para sua montagem.

Durante a busca por novas pesquisas sobre o tema abordado, foi possível adentrar em temáticas diferenciadas conforme as atividades desenvolvidas na farmacovigilância hospitalar. Neste setor, o trabalho precisa ser realizado com eficiência, eficácia e qualidade, em uma prática segura durante as diversas etapas dos processos.

Ao considerar a necessidade de utilização de outros estudos mais aprofundados, a presente pesquisa procurou investir em novos elementos acerca da atuação da equipe de farmácia no âmbito hospitalar, bem como entender as dificuldades encontradas a partir de investigações e notificações das queixas técnicas com base nas RAM's (Reações Adversas a Medicamentos).

Em uma análise do processo de trabalho dos profissionais de farmácia, corroborou-se a importância da farmacovigilância no âmbito hospitalar no intuito de diminuir os riscos ocupacionais, além do alcance da tão almejada valorização profissional. A operacionalização adequada desse setor garante a qualidade da assistência indireta prestada ao paciente, principalmente em atividades relacionadas a detecção, avaliação, compreensão e prevenção dos efeitos adversos a medicamentos.

Portanto, as contribuições pertinentes para a área de farmácia e para a saúde da população de modo geral, perpassa pela atuação do profissional que precisa estar preparado e qualificado para reduzir os riscos relativos à utilização de medicamentos, a partir de um acompanhamento sistemático de ocorrências desses eventos, contribuindo assim para sua diminuição e/ou prevenção.

\section{CONCLUSÃO}

Considerando os estudos apresentados, que serviram de base para os resultados, observa-se uma lacuna entre a teoria e a prática dos profissionais da área de saúde, que necessitam de especialização na área de farmacovigilância. Deste modo, é importante a construção de uma rede colaborativa para a promoção de uma vigilância ativa da segurança dos medicamentos. 
Participante desta rede colaborativa, o farmacêutico necessita manter-se ativo e capacitado, para que o reconhecimento e a identificação das RAM experimentadas pelos pacientes sigam a notificação aos órgãos regulatórios com vistas a promover uma utilização segura e racional dos medicamentos.

Os subsídios para o planejamento de estratégias de sensibilização a respeito da importância das atividades de FV para a identificação das RAMs e a promoção do uso racional de medicamentos voltados ao cuidado do paciente podem ser discutidos com os profissionais da área de saúde.

Deste modo, importa repensar currículos e avaliar a integração ensino-serviço, além de investir em estratégias educativas direcionadas aos profissionais de farmácia, para que se sintam aptos a identificar/avaliar situações de risco e conhecer os sistemas de notificação de incidentes. À guisa de conclusão, as publicações enfatizaram a discussão sobre a Farmacovigilância hospitalar e a atuação do farmacêutico direcionada à investigação e notificação de queixas com base nas RAM's, apresentando um impacto positivo dessa atuação em relação à assistência à saúde, como segurança do paciente. Tais estudos podem fundamentar o processo de tomada de decisão e a formulação de estratégias que visem ampliar a capacidade de gestão e a qualidade do serviço prestado.

\section{REFERÊNCIAS BIBLIOGÁFICAS}

1. Pinheiro, H. C. G; Pepe, V. L. E. Reações adversas a medicamentos: conhecimento e atitudes dos profissionais de saúde em um hospital-sentinela de ensino do Ceará-Brasil, 2008. Epidemiol. Serv. Saúde [online], V. 20, n. 1, p. 57-64, 2011. Disponível em: www.scielo.br. Acesso em: ago 2021.

2. Alomar, M. J. Factors affecting the development of adverse drug reactions. Saudi Pharm J [on line]. 2014. 22(2), p. 83-94. Disponível em: http://dx.doi.org. Acesso em: ago 2021.

3. OMS. Organização Mundial da Saúde. La farmacovigilancia: garantía de seguridad en el uso de los medicamentos, 2004, 6(2): 2-4.

4. Cano, F. G.; Rozenfeld, S. Adverse drug events in hospitals: a systematic review. Cad Saúde Pública 2009; 25 Suppl 3:S360-72. Disponível em: www.scielo.br. Acesso em: ago 2021.

5. Mota, D. M.; Vigo, A.; Kuchenbecker, R. S. Evolução e elementos-chave do sistema de farmacovigilância do Brasil: uma revisão de escopo a partir da criação da Agência Nacional de Vigilância Sanitária. Cad Saúde Pública 2018; 34(10):e00000218. Disponível em: www.scielo.br. Acesso em: ago 2021.

6. OMS. Collaborating Centre for Drug Statistics Methodology. Anatomical Therapeutic Chemical classification system with Defined Daily Doses (ATC/DDD Index), 2006. Disponível em: www.whocc.no/atcddd/. Acesso em: ago 2021. 
7. SINITOX. Sistema Nacional de Informações Tóxico-Farmacológicas. Dados de 2015. Disponível em: http://sinitox.icict.fiocruz.br. Acesso em: ago 2021.

8. Nunes, P. H. C. [et al.]. Intervenção farmacêutica e prevenção de eventos adversos. Revista Brasileira de Ciências Farmacêuticas, V. 44, n. 4, out./dez., 2008. Disponível em: www.scielo.br. Acesso em: ago 2021.

9. Hinrichsen, S. L.; Oliveira, C. L. F.; Campos, M. A.; Possas, L. C. M.; Sabino, G.; Vilella, T. A. S. Gestão da Qualidade e dos riscos na segurança do paciente: estudo-piloto. Revista de Administração Hospitalar e Inovação em Saúde, v. 7, n. 7, p. 10-17, 2011. Disponível em: http://www.spell.org.br. Acesso em: ago 2021.

10. Moscou, K.; Kohler, J. C.; MaGahan, A. Governance and pharmacovigilance in Brazil: a scoping review. J Pharm Policy Pract, 2016 Feb 8;9:3. Disponível em: https://pubmed.ncbi.nlm.nih.gov. Acesso em: ago 2021.

11. Giordani, F. Vigilância de eventos adversos a medicamentos em hospitais: aplicação e desempenho de rastreadores, Rev Bras Epidemiol 2012; 15(3): 455-67; SP, 2012.

12. Margonato, F. B.; Thomson, Z.; Paoliello, M. M. B. Determinantes nas intoxicações medicamentosas agudas na zona urbana de um município do Sul do Brasil. Cadernos de saúde pública. Rio de Janeiro, v. 24, n. 2, p. 333-341, fev, 2008. Disponível em: www.arca.fiocruz.br. Acesso em: ago 2021.

13. Cardinal, L.; Fernandes, C. Intervenção farmacêutica no processo de validação da prescrição médica. Revista e-ciências, v. 4, n. 2, p. 12-19,2016. Disponível em: https://unijuazeiro.edu.br. Acesso em: ago 2021.

14. Romeu, G. A.; Távora, M. R. F.; Costa, A. K. M.; Souza, M. O. B.; Gondim, A. P. S. Notificação de reações adversas em um Hospital Sentinela de Fortaleza - Ceará. R. Bras. Farm. Hosp. Serv. Saúde. São Paulo v.2 n.1 5-9 jan./abr. 2011. Disponível em: www.sbrafh.org.br. Acesso em: ago 2021.

15. Duarte, M. L.; Batista, L. M.; Albuquerque, P. M. S. Notificações de farmacovigilância em um hospital oncológico sentinela da paraíba. Universidade Federal da Paraíba. Rev. Bras. Farm. Hosp. Serv. Saúde. São Paulo, v.5 n.1 7-11 jan./mar. 2014. Disponível em: www.scielo.br. Acesso em: ago 2021.

16. Ferreira Neto, P. T. P.; Simão, R. F. M.; LaCava, A. M. Atividades de farmacovigilância em um hospital pediátrico: uma proposta de melhoria. Revista ACRED - ISSN 2237-5643 v. 4, n. 8, p. 17-26 (2014). Disponível em: https://dianet.unirioja.es. Acesso em: ago 2021.

17. Pezato, T. P. J.; Cesaretti, M. L. R. Farmacovigilância hospitalar: importância do treinamento de profissionais na potencialização de suas ações. Rev. Fac. Ciênc. Méd. Sorocaba, v. 17, n. 3, p. 134-139, 2015. Disponível em: https://revistas.pucsp.br. Acesso em: ago 2021.

18. Modesto, A. C. F.; Ferreira, T. X. A. M.; Provin, M. P.; Amaral, R. G.; Lima, D. M. Reações Adversas a Medicamentos e Farmacovigilância: Conhecimentos e Condutas de Profissionais de Saúde de um Hospital da Rede Sentinela. Revista Brasileira de Educação Médica. 40 (3):401-410. Goiânia, GO, 2016. Disponível em: www.scielo.br. Acesso em: ago 2021.

19. Pereira, L. M. V.; Menezes, J. C,; Vendruscolo, A. C. S. Desenvolvimento de instrumento para avaliação de notificações de suspeita de eventos adversos a medicamentos. Revista 
QualidadeHC. Publicado em 2016, p. 60-69. Disponível em: www.hcrp.usp.br. Acesso em: ago 2021.

20. Fernandes, L. L. A importância do farmacêutico hospitalar juntamente com a equipe multidisciplinar na Unidade de Terapia Intensiva (UTI). Revista Farol. Faculdade Rolim de Moura, Rondônia, V. 8, n. 8, p. 5-21, jun./2019. Disponível em: www.revistafarol.com.br. Acesso em: ago 2021.

21. Ferreira-da-Silva, R.; Ribeiro-Vaz; I.; Silva, A. M.; Marques, J.; Polónia, J. J. Retrospectiva de 20 anos de atividade da Unidade de Farmacovigilância do Porto, Portugal. Cadernos de Saúde Pública. ISSN 1678-4464. 37, n. 10. Rio de Janeiro, Out/2021. Disponível em: http://cadernos.ensp.fiocruz.br. Acesso em: out 2021.

22. Malin, A.; Aso, V.; Johanna, B. [et al.]. A Survey of public knowledge and awareness related to antibiotic use and resistance in Sweden. Journal of Antimicrobial Chemotherapy. Sweden, 2010 .

23. Santos, S. L. F. [et al.]. Papel do farmacêutico no uso racional de antibióticos. RSC online, 2017; 6(1): p. 79-88.

24. Flasche, S.; Atkins, K. E. Balancing benefits and risks of antibiotic use. J. Infect. Dis. 2018 Jun 5. doi: 10.1093/infdis/jiy344. [Epub ahead of print]. Disponível em: https://pebmed.com.br. Acesso em: set 2021.

25. Paula, C. C. S.; Campos, R. B. F.; Souza, M. C. R. F. Uso irracional de medicamentos: uma perspectiva cultural. Brazilian Journal of Development, Curitiba, v.7, n.3, p. 21660-21676 mar 2021. Disponível em: https://www.brazilianjournals.com. Acesso em: set 2021.

26. Dantas, M. L. J.; Ribeiro, P. V. L.; Justino, E. C. [et al.]. A Importância da notificação na Farmacovigilância para a Segurança do Paciente: desafios e Limites. XII Congresso Brasileiro de Farmácia Hospitalar e Serviços de Saúde. Anais, Fortaleza, CE, 23 a 25 de maio de 2019. Disponível em: www.sbrafh.org.br. Acesso em: set 2021.

27. Rodríguez A, Ortega M, Garzón L, et al. Tendencias de los fenotipos de resistencia bacteriana en hospitales públicos y privados de alta complejidad de Colombia. Revista Panam Salud Publica 2011; 30(6):627-33. 